
allemande

50-1| 2018

Regards franco-allemands sur la justice dans la construction européenne | Les élections législatives de 2017 en Allemagne et en Autriche

\title{
La « justice spatiale » : regards français et allemands en géographie et en aménagement
}

Anaïs Volin , Lisa Rolland et Lydia Coudroy de Lille

\section{(2) OpenEdition \\ Journals}

Édition électronique

URL : https://journals.openedition.org/allemagne/629

DOI : 10.4000/allemagne.629

ISSN : 2605-7913

Éditeur

Société d'études allemandes

Édition imprimée

Date de publication : 30 juin 2018

Pagination : $9-25$

ISSN : 0035-0974

Référence électronique

Anaïs Volin , Lisa Rolland et Lydia Coudroy de Lille, « La « justice spatiale » : regards français et allemands en géographie et en aménagement », Revue d'Allemagne et des pays de langue allemande [En ligne], 50-1 | 2018, mis en ligne le 30 juin 2019, consulté le 02 juin 2022. URL : http://

journals.openedition.org/allemagne/629; DOI : https://doi.org/10.4000/allemagne.629 


\section{La «justice spatiale»: regards français et allemands en géographie et en aménagement}

- Anaïs Volin*, Lisa Rolland**, Lydia Coudroy de Lille***

\section{Introduction}

L'association entre justice et espace ne va pas de soi a priori. Longtemps, la géographie s'est pensée comme sciences «des lieux» ou "des paysages» et ne s'y est guère intéressée. Mais en se rapprochant des sciences sociales après la guerre, la géographie humaine a commencé à penser cette articulation sans forcément mobiliser directement le terme de «justice». En France et en Allemagne, ce cheminement n'emprunte pas les mêmes voies: si la justice spatiale et ses déclinaisons semblent éminemment liées aux travaux sur la justice sociale chez les géographes français, la notion est davantage comprise outre-Rhin au regard des inégalités territoriales liées au contexte historique et géopolitique de l'Allemagne. Est-ce dû seulement à l'évolution de la discipline en France et en Allemagne, aux cultures scientifiques et à la structuration institutionnelle de la géographie? Ou bien aussi aux configurations territoriales des deux États, qui ne posent pas les mêmes défis aux chercheurs? Par ailleurs, la notion de justice a été mobilisée, sous cette appellation ou d'autres, dans l'aménagement du territoire, prolongement opérationnel de la géographie. Là aussi, quelles distinctions entre France et Allemagne? Enfin, l'Union européenne (UE) développe des politiques territoriales que l'on peut considérer comme des déclinaisons de la justice spatiale, mais sans jamais emprunter ce terme.

Alors que depuis quelques années la notion de justice revient dans le débat en sciences sociales, puisant dans une bibliographie largement anglophone, cette contribution propose un regard nouveau, centré sur sa place dans la géographie et l'aménagement en France et en Allemagne. Qu'est-ce que la notion de justice a apporté à la géographie? Inversement, en quoi la géographie a-t-elle contribué aux réflexions sur

\footnotetext{
* Doctorante en géographie à l'Université Lumière Lyon 2.

** Docteure en géographie-aménagement, Université Paris Ouest Nanterre La Défense.

*** Professeure de géographie, Université Lumière Lyon 2.
} 
la justice en y introduisant cette dimension spatiale? Dans une première partie, une approche diachronique de la notion de justice spatiale est exposée, avec ses inspirations théoriques, les postures de recherche et les dispositifs méthodologiques qui lui sont propres. La deuxième partie est consacrée aux apports des travaux sur la justice spatiale à la géographie dans les deux pays, aussi bien sur ses objets que sur les terrains d'étude. Enfin la dernière partie met l'accent sur une des déclinaisons majeures de la notion de justice spatiale, celle des disparités régionales, et montre comment elle a inspiré l'aménagement du territoire, français, allemand, mais aussi européen.

\section{Comment la géographie a intégré la justice spatiale}

Dans la géographie française, l'articulation entre la notion de justice spatiale et le thème des inégalités territoriales et socio-spatiales a été introduite à la fin des années 1970 par Paul Claval, qui a coordonné un court dossier de l'Espace géographique sur «Espace et justice spatiale». Celui-ci comprenait une note de David Harvey, un texte de Paul Claval exposant la manière dont les thèses, développées dans La justice sociale et la $v_{i l l e}^{(1)}$ et dans les travaux du géographe britannique David Smith, interpellaient la géographie humaine, ainsi que des réactions de deux autres géographes, Antoine S. Bailly et Renée Rochefort ${ }^{(2)}$. Peu après, Alain Reynaud apporta une contribution plus conséquente, avec Société, espace et justice ${ }^{(3)}$. Il avait déjà une douzaine d'ouvrages à son actif, dans ce qu'on appelle la géographie "économique et régionale», sur des espaces allant de la Lorraine au Mexique, en passant par l'URSS. Cette géographie «tous-terrains», attentive aux relations entre niveaux de développement et aux hétérogénéités spatiales, a peut-être amené son auteur à cette réflexion sur les «inégalités régionales et [la] justice socio-spatiale», sous-titre de l'ouvrage. Entre-temps, il a aussi publié en 1979 un essai sur Le concept de classe socio-spatiale, qui fonde sa réflexion sur la justice socio-spatiale ${ }^{(4)}$. Cette catégorie, définie comme "tout groupe social défini par une appartenance spatiale» ${ }^{(5)}$, lui permet de revisiter les notions classiques en géographie de centre et de périphérie et de parvenir à un questionnement sur l'atténuation des inégalités entre ces derniers. C'est là qu'intervient la «justice socio-spatiale». Inspiré par Tocqueville, mais aussi par des références britanniques ${ }^{(6)}$, par Karl Polanyi et Henri Lefebvre ${ }^{(7)}$, Reynaud définit la justice comme une réduction des inégalités et une égalité d'accès (y compris géographique) à des biens ou des services, notamment urbains. Il postule qu'elle «tend à atténuer les inégalités entre centre et périphérie» mais en devant surmonter deux principaux obstacles attribués par l'auteur à la population. Le premier concerne «l'égoïsme socio-spatial» dont fait preuve la population du centre (accès privilégié à l'emploi et

1 David Harvey, Social Justice and the City, Londres, Hodder Arnold, 1973, 336 p.

2 Paul Claval, «Espace et justice sociale», L’Espace géographique, 7/4 (1978), p. 303-304.

3 Alain Reynaud, Société, espace et justice, Paris, Presses universitaires de France, 1981, 263 p.

4 Alain ReynAuD, «Le concept de classe socio-spatiale: la notion de région dans son contexte social», Travaux de l'Institut de Géographie de Reims, n 38 (1979), p. 17-21.

5 A. Reynaud, Société, espace, justice (note 3), p. 11.

6 Bryan Elis Coates, Ronald John Johnston, Paul Leslie Knox, Geography and Inequality, Oxford, Oxford University Press, 1977, 292 p.; David M. Sмiтн, Human geography: a welfare approach, Londres, Hodder Arnold, 1977, 416 p.

7 Henri Lefebvre, Le droit à la ville, Paris, Anthropos, 1968 (2 éd.), 164 p. 
aux équipements, concentration des activités et des services), "qui refuse de se laisser affaiblir sous prétexte d'aider d'autres classes moins favorisées » ${ }^{(8)}$. Le deuxième est celui des «égoïsmes individuels» des habitants de la périphérie, prompts à jouir de meilleures opportunités ailleurs que dans leur environnement dégradé, notamment dans le centre ${ }^{(9)}$ : meilleur environnement social, main-d'œuvre mieux formée, opportunités d'emploi et de carrière. En outre, Alain Reynaud soupçonne la «charité» de se tapir derrière la justice socio-spatiale, et il en voit le spectre sous trois formes d'interventions: les grands équipements "structurants» susceptibles de devenir des cathédrales dans le désert, les aides financières à la personne au nom du welfare, ou les équipements en périphérie, consentis en réalité dans l'intérêt du centre. C’est pourquoi l'auteur plaide pour une «auto-gestion socio-spatiale», ce qui n'est pas sans faire écho aux mouvements sociaux engagés à cette époque dans une lutte politique contre l'extension du camp militaire sur le plateau du Larzac ou le projet de centrale nucléaire de Plogoff à la pointe du Raz. Pourtant dans son analyse, c'est bien à la puissance publique qu'il revient de jouer le rôle de redistribuer et d'assurer la réciprocité entre centre et périphérie. Il s'intéresse alors à l'aménagement du territoire, et convoque sa vaste culture de géographe «régional» pour décliner les types de relations entre la puissance publique et les périphéries qu'il qualifie d'«ignorées", de "pénalisées» ou d' "assistées» - de sorte que finalement, il en vient à assimiler un idéal (la justice spatiale) avec son vecteur (l'aménagement du territoire). Cet ouvrage pose des jalons théoriques importants, et revendique ce statut.

L'autre source majeure d'inspiration pour les chercheurs francophones est La théorie de la justice de John Rawls ${ }^{(10)}$, dont la première traduction remonte à 1987. Rawls, à vrai dire, n'aborde jamais la dimension spatiale dans cet ouvrage, mais il apporte des arguments solides pour analyser théoriquement et empiriquement les inégalités sociales et spatiales. D’une part, il permet lui aussi de repenser le binôme centre-périphérie, en questionnant la "justice» et l'efficacité de la relation entre ses deux composantes; d'autre part, son œuvre a fourni des assises importantes pour la géographie du développement, par son attention aux notions de liberté, de contrat, et ses considérations sur l'esclavage. C'est pourquoi il constitue une référence dans une partie de la géographie des Suds. Enfin il conforte les politiques territoriales œuvrant dans le sens de la compensation des handicaps structurels: "le principe de différence donne un certain poids aux arguments mis en évidence par le principe de réparation, à savoir que les inégalités non méritées doivent être corrigées» (p. 131). Autrement dit, Rawls fournit un socle théorique aux dispositifs de géographie prioritaire, si l'on transfère ses considérations du social au spatial.

En Allemagne, les travaux sur la justice sont initialement menés en philosophie et en sciences politiques dès les années 1960. La référence à l'espace y est absente, ces travaux s'intéressant d'abord à la justice procédurale (Verfahrensgerechtigkeit). Les théories de l'équité de John S. Adams ${ }^{(11)}$ servent tout d'abord de cadre de pensée,

\footnotetext{
8 A. Reynaud, Société, espace, justice (note 3), p. 111.

9 Ibid., p. 120.

10 John Rawls, Théorie de la justice (A Theory of Justice, 1971), Paris, Le Seuil, 1987, 666 p.

11 John S. Adams, "Inequity in social exchange», Journal of Abnormal and Social Psychology, $\mathrm{n}^{\circ} 62$ (1965), p. 335-343.
} 
articulant échelles individuelle et sociétale dans la compréhension de dynamiques de sanctions, de coopérations ou encore d'établissement de règles. Dans les années 1980, la psychologie s'empare de la question avec Gerald S. Leventhal ${ }^{(12)}$, qui établit six principes de la Verfahrensgerechtigkeit: la cohérence (Konsistenz) - chacun est traité d'égal à égal; l'impartialité (Unvoreingenommenheit) - chaque point de vue est pris en considération; l'exactitude (Genauigkeit) - informations complètes et collecte de données précises; la corrigibilité (Korrigierarbeit) - l'acceptation de l'inconfort; la représentativité (Repräsentativität) - la considération de tous les intérêts; et l'éthique (ethische Rechtfertigung) - la justification des jugements moraux ${ }^{(13)}$.

Les géographes allemands se sont emparés de la dimension spatiale de la justice tout récemment. Ils s'inspirent essentiellement du courant de la new cultural geography (David Harvey) mais aussi des travaux d'Henri Lefebvre ou de Michel Foucault ${ }^{(14)}$. La Neue Kulturgeographie (2000), la nouvelle géographie culturelle, renoue avec des lectures marxistes interrompues pendant la guerre froide et la partition de l'Allemagne. Le projet de cette géographie allemande cherche moins à adopter un point de vue critique dans la discipline qu'à remettre au cœur de la pensée géographique la construction matérialiste et historique des faits sociaux développée par Karl Marx. En effet, la géographie germanophone de la RFA, à la différence du monde francophone et anglophone, a délaissé la posture critique ${ }^{(15)}$, comme les approches critiques germanophones ont délaissé l'espace $^{(16)}$. Au-delà des géographes, Karl Marx et Henri Lefebvre ${ }^{(17)}$ sont alors convoqués pour défendre une vision de l'espace dans ses significations mais aussi dans sa matérialité: les formes de domination que constituent le capitalisme via l'État et les institutions sont marquées dans l'espace à travers l'aménagement du territoire. À cet égard, des travaux récents ${ }^{(18)} s^{\prime}$ intéressent aux instruments de contrôle des individus (outils, moyens de sécurité), largement étudiés par ailleurs dans les travaux anglo-saxons ${ }^{(19)}$.

Par-delà les situations spécifiques de la recherche et de structuration des disciplines en France et en Allemagne, les positionnements théoriques et méthodologiques des travaux sur la justice spatiale sont généralement orientés par une critique du système libéral et par un engagement du chercheur sur son terrain et face à celui-ci. Deux

12 Gerald S. Leventhal, "What should be done with equity theory?», Social Exchange, n 1 (1980), p. 27-55.

13 Aujourd'hui, l'emploi du terme de Verfahrensgerechtigkeit est davantage usité par les économistes pour désigner la justice procédurale et plus spécifiquement pour traiter les problématiques d'équité.

14 Michel Foucault, L'ordre du discours, Paris, Gallimard, 1971, 84 p.

15 Mélina Germes, Georg Glasze, Florian Weber, «Neue Kulturgeographie - débats et perspectives de la nouvelle géographie culturelle germanophone», Cybergeo, 2011, p. 1-20.

16 L'attention portée - à nouveau - à l'espace dans la géographie allemande est très récente du fait d'un contexte historique particulier (nazisme, guerre froide, partition de l'État). Celui-ci a davantage laissé la place à l'histoire, notamment l'histoire politique et sociale, qu'à la géographie et à la compréhension de l'espace. Lire à ce sujet: Hélène MiArd-Delacroix, Guillaume GArner, Béatrice von Hirschinausen (dir.), Espaces de pouvoir, espaces d'autonomie en Allemagne, Lille/Villeneuve d'Ascq, Septentrion, 2010, 259 p.

17 H. Lefebvre, Le droit à la ville (note 7).

18 Bernd Belina, Raum, Überwachung, Kontrolle: vom staatlichen Zugriff auf städtische Bevölkerung, Münster, Westfälisches Dampfboot, 2006, 321 p.

19 D. Harvey, Social Justice and the City (note 1). 
courants peuvent être dégagés: un premier, hérité des bouleversements de la discipline dans les années 1970, rattaché à la géographie critique ou radicale, contient une forte ambition militante. L'analyse dialectique, la justice sociale et l'égalité socio-spatiale en constituent les trois grilles de lecture principales. Développé aux États-Unis autour des travaux fondateurs d'Edward Soja ${ }^{(20)}$, ce courant renouvelle aujourd'hui la géographie, aussi bien en France qu'en Allemagne, dans un contexte international de regain des approches critiques en sciences sociales. En France, les théories de Henri Lefebvre sur le «droit à la ville» ${ }^{(21)}$ et de David Harvey ${ }^{(22)}$ sont reprises pour dénoncer les politiques néolibérales ou libérales à l'origine d'inégalités et de disparités territoriales ${ }^{(23)}$. Pour certains, la notion de justice spatiale constitue alors un levier théorique pour interroger l'aménagement du territoire et ses acteurs ${ }^{(24)}$. En Allemagne (ou à propos de celle-ci), cette grille de lecture néo-marxiste est mobilisée pour relire l'unification allemande au sein des sciences du territoire ${ }^{(25)}$. La justice spatiale est convoquée pour étudier sous un nouvel angle le "déclin» urbain et régional, la "périphérisation » et la marginalisation $^{(26)}$ des nouveaux Länder orientaux dans les études est-allemandes ${ }^{(27)}$ (Ostdeutschlandforschung). Les courants les plus critiques mettent en exergue les logiques néolibérales et conservatrices de la réunification pour dénoncer à la fois des processus de privatisation des anciens biens nationalisés comme les usines, les mines, mais aussi les logiques de pouvoir et la dépendance des petites et moyennes villes aux échelons territoriaux supérieurs et aux acteurs économiques ${ }^{(28)}$.

Un second courant mobilise la notion de justice spatiale du côté de la géographie du développement et de l'environnement. En France comme en Allemagne, des auteurs, empruntant à John Rawls, cherchent à articuler justice, développement et équité, suggérant une organisation optimale de l'espace qui garantirait à tous un accès égal aux ressources ${ }^{(29)}$. Il ne s'agit pas d'étudier la justice spatiale «comme une justice entre les lieux, mais comme la dimension spatiale de la justice entre les hommes ${ }^{\left({ }^{(3)}\right.}$, autrement

20 Edward SojA, Seeking spatial justice, Minneapolis, University of Minnesota Press, 2010, 288 p.

21 H. Lefebvre, Le droit à la ville (note 7).

22 D. Harvey, Social Justice and the City (note 1).

23 Andy Merrifield, Erik Swyngedouw, The Urbanization of Injustice, Londres, Lawrence and Wishart, 1996, 256 p.

24 Frédéric Dufaux, Pascale Philifert, Justice spatiale et politiques territoriales, Paris, Presses universitaires de Paris Ouest, 2013, 322 p.

25 Christian Schulz, "Les sciences du territoire en Allemagne», CIST2011 - Fonder les sciences du territoire $1^{\text {er }}$ colloque du CIST, Paris, 2011, p. 430-436.

26 Hélène Rотн, «Du déclin à la périphérisation: quand les courants constructivistes et critiques revisitent les différenciations spatiales en Allemagne», Cybergeo: European Journal of Geography, n 758 (2016), p. 1.

27 Jusque-là plutôt en marge dans le système académique.

28 Manfred KüHn, Matthias Bernt, «Peripheralization and Power», in: Andrea Fischer-TAhir, Matthias Naumann (éd.), Peripheralization: The Making of Spatial Dependencies and Social Injustice, Wiesbaden, Springer, 2013, p. 302-316.

29 Bernard Bret, Pour une géographie du Juste. Lire les territoires à la lumière de la philosophie morale de John Rawls, Paris, Presses universitaires de Paris Ouest, 2015, 322 p.; Ulf Hahne, Jan M. Stielike, «Gleichwertigkeit der Lebensverhältnisse - Zum Wandel der Normierung räumlicher Gerechtigkeit in der Bundesrepublik Deutschland und der Europäischen Union ", Ethik und Gesellschaft, n 1 (2013), 40 p.

30 Bernard BRET, «Notions à la une: justice spatiale», Géoconfluences, 2015, mis en ligne le 18.09.2015, consulté le 19.12.2017. 
dit l'idéal de justice spatiale ne promet pas l'égalité de la répartition des ressources, mais l'équité dans l'accès à celles-ci entre les individus. Ces travaux cherchent à comprendre, d'une part la construction d'inégalités sociales au prisme de la justice environnementale, basées sur la dissociation spatiale entre « enfers gris et paradis verts » ${ }^{(31)}$, d'autre part l'accès aux services environnementaux ainsi qu'aux ressources ${ }^{(32)}$.

Les méthodes employées découlent de postures de recherche ancrées dans le mouvement post-moderne, et parfois post-colonial, non surplombantes par rapport au terrain et ses acteurs: elles posent de manière explicite les enjeux politiques de la recherche sur le réel. Il faut dire que beaucoup, surtout parmi les géographes français, ont «fait leurs classes» dans des terrains sensibles, comme l'Afrique du Sud. La géographie radicale ou critique a donc fait grandement évoluer la discipline vers des méthodes participatives $^{(33)}$. Des modalités de restitution originales par ailleurs répandues dans la géographie post-moderne s'y développent: ces nouvelles méthodes ne sont certes pas propres aux tenants de la justice spatiale mais ils en font un usage systématique en cherchant à intégrer les acteurs locaux et les groupes concernés par les enjeux de justice. Les chercheurs utilisent par exemple la vidéo ${ }^{(34)}$, présentent leurs résultats devant les groupes qu'ils étudient, afin de co-construire avec eux la recherche ${ }^{(35)}$.

Dans le panorama de la recherche internationale, la géographie française est emblématique du renouveau des travaux sur la notion de justice spatiale. En effet, en 2008, un colloque "Justice et injustice spatiales» fut organisé à l'Université Paris Ouest Nanterre La Défense (dans l'amphithéâtre «Henri Lefebvre»...), comptant parmi ses invités le géographe américain Edward Soja, lui-même passeur de Lefebvre aux ÉtatsUnis. Cet événement conduisit à la publication d'un ouvrage ouvrant la structuration de ce champ de recherche en France ${ }^{(36)}$, mais aussi à l'édition d'un numéro spécial des Annales de Géographie, et à la création d'un support de revue en ligne ad hoc, bilingue (français et anglais) et international, la revue justice spatiale/spatial justice, qui ne trouve pas d'équivalent en Allemagne.

Ces quelques jalons des ancrages théoriques et des approches de la justice chez les géographes français et allemands montrent une empreinte néo-marxiste et anglosaxonne commune aujourd'hui, et un enracinement plus ancien côté français. De part et d'autre du Rhin, la notion de justice spatiale a renouvelé l'approche des inégalités et des disparités spatiales, qu'elles prennent forme entre individus ou groupes sociaux, et a suscité des dispositifs méthodologiques ad hoc.

31 David Blanchon et al., "Comprendre et construire la justice environnementale », Annales de géographie, n 665-666 (2009), p. 36.

32 Mathieu DurAnd, Sylvy JAGLIN, «Inégalités environnementales et écologiques: quelles applications dans les territoires et les services urbains?», Flux, n 89-90 (2012), p. 4-14; Gabriele Bolte et al., Umweltgerechtigkeit, Chancengleichheit bei Umwelt und Gesundheit: Konzepte, Datenlage und Handlungsperspektiven, Berne, Verlag Hans Huber, 2012, 440 p. Béatrice Collignon, «L'éthique et le terrain», L’information géographique, nº 1 (2010), p. 63-83. Chloé Buire, «Films de terrain: pratique, réflexivité et écriture. Expérience d’une géographe utilisant l'outil vidéo ", in: Sandra Breux, Jean-Pierre Collin, Catherine Gingras (dir.), Représenter l'urbain: apports et méthodes, Laval, Presses universitaires de Laval, 2014, $424 \mathrm{p}$.

35 Marie Chabrol, De nouvelles formes de gentrification? Dynamiques résidentielles et commerciales à Château-Rouge, thèse de doctorat de géographie, Université de Poitiers, 2012, 405 p. 


\section{Définitions et déclinaisons de la justice spatiale en géographie}

Cette généalogie partiellement commune peut laisser penser que la justice spatiale est définie dans les deux langues et dans la discipline géographique de manière consensuelle, ce qui sera abordé dans cette deuxième partie. En outre, elle interroge les déclinaisons empiriques de la notion: à quels objets a-t-elle été appliquée, et dans quels terrains, en France et en Allemagne?

Les auteurs du numéro spécial des Annales de géographie ${ }^{(37)}$ (mai 2009) et du premier numéro de la revue justice spatiale/spatial justice ${ }^{(38)}$ (septembre 2009) sont très prudents dès qu'il s'agit de définir la justice spatiale. Les deux éditoriaux s'accordent sur la bivalence de celle-ci: ils lui attribuent une dimension structurelle lorsqu'elle " conduit à se poser des questions sur les distributions spatiales (de biens, de services, de personnes...) » ${ }^{(39)}$ ou bien une dimension procédurale lorsqu'on "est conduit à se poser des questions de représentations de l'espace, d'identités (territoriales ou non), de pratiques ${ }^{(40)}$. En 2013, Frédéric Dejean s'essaie à une synthèse des définitions de la justice spatiale et constate que «la recherche récente [...] ne lui confère pas un statut épistémologique clair» et se résigne à y voir une approche, une thématique ou un outil au service d'objets et de problématiques variés ${ }^{(41)}$. De fait, l'entrée «justice spatiale» dans les dictionnaires de géographie français reste discrète. Roger Brunet, dans Les mots de la géographie (1992), reprend les travaux d'Alain Reynaud, et la définit comme une «forme de justice sociale liée premièrement à la compensation relative des inégalités d'accès aux équipements et autres avantages comparés des lieux [...] deuxièmement à la compensation des disparités territoriales » ${ }^{(42)}$. Plus récemment, seul Le Dictionnaire de l'espace et des sociétés ${ }^{(43)}$ en propose depuis 2013 une définition englobante en tant que «dimension spatiale de l'idée, des conceptions et des pratiques de la justice».

Du côté allemand, pour désigner des disparités d'ordre spatial, le dictionnaire Diercke - Wörterbuch allgemeine Geographie ${ }^{(44)}$ parle de Raumdisparitäten ou räumliche Disparitäten (disparités territoriales). Contrairement au français, le lexique allemand s'attarde tout d'abord sur les disparités territoriales avant de s'intéresser à la justice spatiale. L'entrée räumliche Disparitäten est définie dans le Lexikon der Geographie $^{(45)}$ de 2001 comme: «Disparitäten innerhalb einer Region; ungleiche Ausstattung eines Raumes mit Arbeitsplätzen, Dienstleistungen, Infrastruktur

37 Philippe Gervais-Lambony, Frédéric Dufaux, «Justice... spatiale!», Annales de géographie, 1-2 (2009), n 665-666, p. 3-15

38 Frédéric Dufaux et al., «n 01. Avis de naissance», justice spatiale/spatial justice, $\mathrm{n}^{\circ} 1$, septembre 2009.

39 Gervais-lambony/Dufaux, «Justice... spatiale!» (note 37).

40 Dufaux et al., « $\mathrm{n}^{\circ} 01$. Avis de naissance» (note 38 ).

41 Frédéric DEJEAN, «État de la littérature. La “justice spatiale” : revue des savoirs francophones et anglophones », Critiques internationales, $\mathrm{n}^{\circ} 4$ (2013), p. 171-183.

42 Roger Brunet et al., Les mots de la géographie, dictionnaire critique, Paris, Reclus, 1992, p. 289.

43 Jacques LÉvy, Michel Lussault, Dictionnaire de la géographie et de l'espace des sociétés, Paris, Belin, 2013, p. $582-585$.

44 Dictionnaire général de géographie, Helmut Leser (éd.), Diercke Wörterbuch allgemeine Geographie, Munich, Deutscher Taschenbuch Verlag, 1997 (12 éd.), 1037 p.

45 Ernst Brunotte et al., Lexikon der Geographie, Heidelberg/Berlin, Spektrum Akademischer Verlag, 2001, 5 volumes. 
sowie sozioökonomischen und demographischen Merkmalen» ${ }^{(46)}$. Les plus proches traductions recensées de la justice spatiale sont Verfahrensgerechtigkeit et Verteilungsgerechtigkeit et les travaux restent peu nombreux à ce sujet. Ces deux notions ne renvoient pas directement à l'espace, mais à deux conceptions de la justice ayant des implications spatiales, soit la justice procédurale (Verfahren), qui s’intéresse aux dangers sociaux et environnementaux dans un souci d'équité (cf. supra partie 1), soit à son résultat, ou à une solution, celle de la répartition (Verteilung). On retrouve ici la distinction faite par les géographes français dans l'éditorial du premier numéro de la revue justice spatiale/spatial justice entre dimensions structurelle et procédurale de la justice spatiale. Par ailleurs, des travaux récents proposent le terme de raümliche Gerechtigkeit pour définir la justice entre des territoires, et entre des territoires et les Hommes puisque ces espaces sont produits socialement et politiquement ${ }^{(47)}$. Le numéro 1 de la revue Ethik und Gesellschaft (2013) consacré à «Der spatial Turn der sozialen Gerechtigkeit» interroge d'ailleurs la notion de justice spatiale en ce sens: «wie relevant das Konzept der Gerechtigkeit für die gesellschaftliche Produktion von Räumen und das Nachdenken darüber ist» (quelle est la pertinence de la notion de justice pour la fabrique sociale de territoires et la théorisation à propos de celleci ?) ${ }^{(48)}$. C’est précisément cette expression de raümliche Gerechtigkeit que Le Dictionnaire de la géographie et de l'espace des sociétés ${ }^{(49)}$ choisit pour traduire "justice spatiale». Pourtant, si la räumliche Gerechtigkeit fait consensus dans de nombreux travaux récents ${ }^{(50)}$, les chercheurs se sont davantage intéressés jusque-là à l'Umweltgerechtigkeit $^{(51)}$ (justice environnementale) ou bien à l'ökologische Gerechtigkeit ${ }^{(52)}$ (justice écologique).

Ce flou définitoire, la fréquente association entre justice et injustices (ou inégalités) spatiales, l'utilisation de la justice spatiale plutôt comme une approche, amènent logiquement à sa mobilisation au service d'objets variés, en France et en Allemagne. Comme en témoignent des thèses récentes ou des ouvrages collectifs, la notion de justice est étudiée à travers le prisme des inégalités ${ }^{(53)}$ pour s'intéresser aux enjeux

46 «Disparités au sein d’une région; inégalités territoriales en termes d'emplois, services, infrastructures ainsi que des caractéristiques socio-économiques et démographiques.»

47 Martin Schneider, Raum-Mensch-Gerechtigkeit, Paderborn, Schöningh Verlag, 2012, 726 p.

48 Hahne/Stielike, «Gleichwertigkeit der Lebensverhältnisse » (note 29).

49 Lévy/Lussault, Dictionnaire de la géographie et de l'espace des sociétés (note 43).

50 Anne Volkmann, «Sozialräumliche Benachteiligung unter Schrumpfungsbedingungen», in: Uwe Altrock, Ronald Kunze (éd.), Stadterneuerung und Armut. Jahrbuch Stadterneuerung 2016, Wiesbaden, Springer Verlag, 2017, 401 p.; Marc RedePENNING, «Varianten raumbezogener sozialer Gerechtigkeit. Ein sozialgeographischer Versuch über das Verhältnis von Raum und Gerechtigkeit und ein Nachdenken über die Frage was soll wo sein?», Ethik und Gesellschaft, nº 1 (2013), p. 1-28; HAHNE/ STIELIKe, «Gleichwertigkeit der Lebensverhältnisse» (note 29).

51 David Schlosberg, Defining Environmental Justice. Theories, Movements, and Nature, Oxford, Oxford University Press, 2007, 238 p.; Bolte et al., Umweltgerechtigkeit, Chancengleichheit bei Umwelt und Gesundheit (note 32).

52 Anton LeIst, «Ökologische Gerechtigkeit als bessere Nachhaltigkeit», Politik und Zeitgeschichte, $\mathrm{n}^{\circ} 24$ (2007), p. 3-10.

53 Karine Ginisty, Inégalités et (in)justices spatiales à Maputo: pratiques des services urbains (Mozambique), thèse de doctorat de géographie, Nanterre, Université Paris Ouest Nanterre La Défense, 2014. 
de sécurité, de ségrégation ${ }^{(54)}$, de séparation ${ }^{(55)}$, de pouvoir ${ }^{(56)}$ ou encore du droit à la ville $^{(57)}$ : à travers ces processus socio-spatiaux, c'est l'absence de justice et les inégalités consécutives qui sont discutées, voire dénoncées, au risque d'induire un jugement moral entre le juste et l'injuste ${ }^{(58)}$. Ces travaux visent donc à comprendre et analyser l'accès inégal aux aménités étudiées: le logement (notamment social), les services urbains en réseau (l'eau), les espaces publics, l'alimentation ${ }^{(59)}$, les emplois (en Allemagne), etc. D'autres travaux ont pour objet non pas des biens ou services, mais des revendications sociales pour des procédures spatiales plus justes (les découpages territoriaux); d'autres encore portent sur certains acteurs sociaux eux-mêmes et sur leur rapport à l'espace (les femmes, les enfants). Dans le champ des travaux sur la justice spatiale, un traitement particulier est réservé à l'environnement ${ }^{(60)}$ dont la thématique est bien développée dans les deux pays ${ }^{(61)}$. Les travaux allemands privilégient les liens systémiques entre social et environnemental, en s'inspirant des écrits de Robert Bullard sur l'environmental justice ${ }^{(62)}$. Les chercheurs défendent une approche engagée et vont jusqu'à proposer des solutions ou des pistes de réflexion en faveur d'une institutionnalisation de la notion environmental justice dans les politiques publiques. L'environnement est alors compris au sens de "nature» notamment dans les travaux sur l'ökologische Gerechtigkeit (justice écologique) qui traite par exemple de l'égalité des êtres humains et des animaux, dont se réclament certains mouvements écologiques citoyens. Le champ de la justice environnementale, Umweltgerechtigkeit, est quant à lui plus développé et s'intéresse aux lieux de la justice environnementale ainsi qu'à l'égalité des chances par l'accès équitable à la santé(63).

Le tableau 1 présente le champ lexical de la justice spatiale en français et en allemand, dans les sciences géographiques et du territoire. Cette synthèse ne prétend pas rendre

54 Annette Harth, Gitta Scheller, Wulf Tessin (éd.), Stadt und soziale Ungleichheit, Leverkusen, Leske und Budrich Verlag, 2000, 321 p.

55 Pauline Guinard, Johannesburg: l'art d'inventer une ville, Rennes, Presses universitaires de Rennes, 2014, 326 p.

56 Marie Gibert, Juliette Segard, «L'aménagement urbain au Vietnam, vecteur d’un autoritarisme forcé», justice spatiale/spatial justice, $\mathrm{n}^{\circ} 8$ (2015), en ligne.

57 Chloé Buire, À travers pratiques citadines et tactiques citoyennes, la production du droit à la ville au Cap, thèse de doctorat de géographie, Université Paris Ouest Nanterre La Défense, 2011, 434 p.

58 BRET et al., Justice et injustices spatiales (note 36).

59 Camille Hochedez, Julie Le Gall (coord.), «Justice alimentaire», justice spatiale/spatial justice, $\mathrm{n}^{\circ} 9$, janvier 2016; Flaminia PADDEU, «L'agriculture urbaine dans les quartiers défavorisés de la métropole newyorkaise: la justice alimentaire à l'épreuve de la justice sociale», VertigO - la revue électronique en sciences de l'environnement (en ligne), 12/2 (2012), consulté le 19 décembre 2017, http://vertigo.revues.org/1268; Frédéric LANDy, Un milliard à nourrir. Grain, territoire et politiques en Inde, Paris, Belin, 2006, 270 p.

60 En dehors des sciences sociales, les travaux de Catherine Larrère sur la justice environnementale marquent la discipline philosophique, lire à ce sujet, entre autres, Catherine LARRÈrE, «La justice environnementale», Multitudes, n 36 (2009), p. 156-162.

61 David Blanchon, Jean Gardin, Sophie Moreau (dir.), Justice et injustices environnementales, Presses universitaires de Paris Ouest, 2011, 232 p.

62 Robert Bullard, The Quest for Environmental Justice: Human Rights, and the Politics of Pollution, San Francisco, Sierra Club Books, 2005, 414 p.

63 Bolte et al., Umweltgerechtigkeit, Chancengleichheit bei Umwelt und Gesundheit (note 32). 
compte de l'ensemble des travaux de recherche en géographie et en aménagement ${ }^{(64)}$ dans les deux pays, du fait de la structuration différente des disciplines de part et d'autre du Rhin. Les chercheurs présentés dans le tableau sont géographes (principalement côté français), aménageurs, de profils disciplinaires variés (sociologues de l'urbain, politistes...), attestant de la diversité des disciplines aux prises avec ce sujet. Nous proposons une lecture chronologique de l'évolution de la notion de justice spatiale en français et en allemand. Le tableau illustre tout d'abord l'enracinement plus ancien de la notion dans la langue française (à la suite des travaux d'Henri Lefebvre) et son apparition plus tardive, vers les années 2000, dans les travaux allemands. En outre, on observe des deux côtés une thématisation généralisée de la notion au XXI ${ }^{\mathrm{e}}$ siècle, mettant l'accent, notamment en Allemagne, sur les déclinaisons environnementales et écologiques de la justice. Cela peut s'expliquer par une plus grande diversité terminologique en allemand. Dans cette langue, en effet, des variantes existent autour de la notion de justice spatiale, et l'usage du terme de disparités spatiales (Raumdisparitäten ou räumliche Disparitäten) est antérieur à celui de justice spatiale (räumliche Gerechtigkeit).

Tableau 1: La justice spatiale et ses déclinaisons en géographie et aménagement, regards en France et en Allemagne (conception et réalisation: L. Rolland, A. Volin, 2017)

\begin{tabular}{|c|c|c|c|}
\hline & $\begin{array}{c}\text { Déclinaisons } \\
\text { de la justice spatiale }\end{array}$ & Dates-clés & Chercheurs majeurs \\
\hline \multirow{4}{*}{ 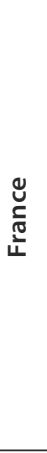 } & $\begin{array}{c}\text { «justice et injustice } \\
\text { spatiale» }\end{array}$ & $\begin{array}{l}1968 \\
1981 \\
2009 \\
2010 \\
2013\end{array}$ & $\begin{array}{c}\text { Henri Lefebvre } \\
\text { Antoine Bailly } \\
\text { Philippe Gervais-Lambony } \\
\text { Bernard Bret } \\
\text { Jacques Lévy }\end{array}$ \\
\hline & «justice socio-spatiale» & 1981 & Alain Reynaud \\
\hline & $\begin{array}{l}\text { «justice et injustice } \\
\text { environnementale» }\end{array}$ & $\begin{array}{c}2009 \\
2009 \\
2009 \text { et } 2011\end{array}$ & $\begin{array}{c}\text { Catherine Larrère } \\
\text { David Blanchon, Jean Gardin } \\
\text { Sophie Moreau }\end{array}$ \\
\hline & «justice alimentaire» & $\begin{array}{l}2012 \\
2016\end{array}$ & $\begin{array}{c}\text { Flaminia Paddeu } \\
\text { Camille Hochedez } \\
\text { Julie Le Gall }\end{array}$ \\
\hline \multirow{2}{*}{ 竞 } & «räumliche Gerechtigkeit» & $\begin{array}{l}2013 \\
2013 \\
2017\end{array}$ & $\begin{array}{c}\text { Ulf Hahne, Jan Stielike } \\
\text { Marc Redepenning } \\
\text { Anne Volkmann }\end{array}$ \\
\hline & «Umweltgerechtigkeit» & $\begin{array}{l}2007 \\
2012\end{array}$ & $\begin{array}{l}\text { David Schlosberg } \\
\text { Gabriele Bolte } \\
\text { Christiane Bunge } \\
\text { Heike Köckler }\end{array}$ \\
\hline
\end{tabular}

Les terrains de recherche de la justice spatiale ciblent en grande majorité la ville, parfois les quartiers, la ville (néolibérale) étant lue comme un cadre producteur d'inégalités et d'injustices vécues et subies par des groupes sociaux. L'influence du «droit à la ville» de Lefebvre est ici évidente. Un grand nombre de géographes français se sont tournés vers les espaces urbains des Suds, notamment en Afrique du Sud ou en 
Amérique latine. Ces choix de terrains peuvent se comprendre aisément au regard d'une lecture inégalitaire de l'espace-monde. Toutefois, de plus en plus de recherches se réorientent vers les Nords et les problématiques de gentrification ${ }^{(65)}$, de fragmentation intra-urbaine, autour des travaux fondateurs, à nouveau anglo-saxons, sur la ville juste ${ }^{(66)}$. Elles permettent de questionner, en France, un des fondements des politiques de la ville (une ville mixte est-elle juste?) ${ }^{(67)}$. En Allemagne, les terrains européens, nationaux et même régionaux sont mis à l'honneur, dans la continuité logique des postures de la nouvelle géographie sociale allemande attentive aux bouleversements de l'espace allemand après 1990. Là aussi, on retrouve le thème du logement, mais également du chômage, qui servent à étudier des logiques de ségrégation territoriale entre Est et Ouest, ou à l'échelle intra-urbaine ${ }^{(68)}$. L'originalité des travaux allemands porte sur l'utilisation privilégiée du thème des inégalités territoriales et des injustices spatiales pour revisiter les effets de la réunification allemande. Les questionnements sur la gentrification ont concerné Berlin en priorité ${ }^{(69)}$. L'espace rural n'est toutefois pas délaissé: outre un numéro spécial de la revue justice spatiale/spatial justice consacré au «droit au village» ${ }^{(70)}$, on peut citer les travaux de ruralistes abordant cette approche de la justice spatiale au Nord comme au Sud, à propos de l'accès à la terre, ou de la production agricole ${ }^{(71)}$.

On remarque à travers cette recension que la notion de justice spatiale a servi de grille de lecture, déclinée sur une palette de plus en plus large d'objets et de terrains d'études. Néanmoins, c'est dans le domaine des disparités territoriales et des moyens engagés pour les résorber qu'elle a le plus inspiré des politiques publiques et qu'elle mérite d'être questionnée, autrement dit dans le domaine de l'aménagement du territoire.

65 Jean-Yves Authier, «Formes et processus de ségrégation dans les quartiers anciens centraux réhabilités. L’exemple du quartier Saint-Georges à Lyon", Sociétés Contemporaines, n 22-23 (1995), p. 107126; Catherine BIDOU-ZACHARIASEN (dir.), Retours en ville - des processus de "gentrification" urbaine aux politiques de "revitalisation" des centres, Paris, Descartes et Cie, 2003, 267 p.; Anne Clerval, La gentrification à Paris intra-muros: dynamiques spatiales, rapports sociaux et politiques publiques, thèse de doctorat en géographie, Université de Paris 1, 2008, 602 p.

66 Susan Fainstein, The just city, New-York, Cornell University Press, 2011, 224 p.

67 Sonia Lehman Frisch, «La ségrégation: une injustice spatiale? Questions de recherche», Annales de géographie, $\mathrm{n}^{\circ}$ 1-2 (2009), p. 94-115.

68 Carsten Grosse Starman, Kerstin Schmidt, "Soziale Segregation lokal gestalten», in: Bertelsmann Stiftung (éd.), Demographie konkret - Soziale Segregation in deutschen Großstädten. Daten und Handlungskonzepte für eine integrative Stadtpolitik, Gütersloh, Bertelsmann Stiftung, 2008, p. 6-9; Klaus FriedriCH, «Gentrifizierung. Theoretische Ansätze und Anwendung auf Städte in den neuen Ländern», Geographische Rundschau, n 52 (2000), p. 34-39; Andrej Holm, Die Restrukturierung des Raumes. Stadterneuerung der 90er Jahre in Ostberlin. Interessen und Machtverhältnisse, Bielefeld, Transcript Verlag, 2006, 356 p.

69 Christian Krajewski, Urbane Transformationsprozesse in zentrumsnahen Stadtquartieren - Gentrifizierung und innere Differenzierung am Beispiel der Spandauer Vorstadt und der Rosenthaler Vorstadt in Berlin, Münster, Universität Münster Institut Geographie, 2006, 356 p.

70 Frédéric Landy, Sophie Moreau (coord.), «Le droit au village», justice spatiale/spatial justice, $n^{\circ} 7$, janvier 2015.

71 Hochedez/Le Gall (coord.), «Justice alimentaire» (note 59). 


\section{Justice spatiale et aménagement du territoire}

Comment l'aménagement du territoire, en France, en Allemagne et dans l'Union européenne, s'est-il emparé du corpus théorique de la justice spatiale pour transformer ou inspirer ses pratiques? À cet égard, la revue justice spatiale/spatial justice ${ }^{(72)}$ (2009) affirme que «la justice spatiale est l'objectif de toutes les politiques d'action sur les territoires». Qu'en est-il de part et d'autre du Rhin mais aussi à l'échelle de l'Union européenne? Il n'est pas question ici d'évaluer les résultats de ces politiques, mais d'interroger comment celles-ci intègrent l'objectif, du moins affiché, de l'égalité territoriale, mobilisent les concepts scientifiques, ou en construisent de nouveaux.

L'aménagement du territoire en France s'est souvent pensé en tension entre une recherche de performance, ou de "compétitivité» (qui amène à soutenir les territoires bien insérés et producteurs de richesses) et de "réparation» envers des régions souffrant de désavantages structurels (insularité, montagne, enclavement) ou conjoncturels (reconversion industrielle). Les dispositifs aménagistes se réclamant du deuxième principe se déclinent en de multiples zonages dans une optique de géographie dite «prioritaire» comme les zones d'adaptation industrielle dans les années 1960 ou les zones franches urbaines dans les années 2000. Ils sont destinés à diriger la création d'emplois dans des régions désavantagées, ou au contraire à la détourner de celles qui en polarisent trop, à commencer par la région parisienne. La logique réparatrice, si elle ne se réclame pas explicitement de la justice spatiale, agit néanmoins en fonction du principe rawlsien de correction et/ou de réparation (de processus ou de phénomènes vus comme des injustices). Ces deux logiques apparemment contradictoires n'apparaissent pas de manière alternée, mais souvent en parallèle, de manière croisée dans l'aménagement du territoire, car celui-ci se pense en réalité simultanément à plusieurs échelles. À une échelle internationale ou globale, l'État valorise ses métropoles et ses régions fortes (la région parisienne notamment). Mais à l'échelle nationale, nul n'a intérêt à voir se développer des enclaves de pauvreté et de déclin, et c'est là qu'intervient la logique de correction des inégalités territoriales.

Comme le montre l'article de Guy Baudelle et Klaus Kunzmann, les configurations territoriales diffèrent totalement entre les deux pays étudiés: c'est pourquoi il en va de même pour les politiques aménagistes et la manière de se saisir de l'espace ${ }^{(73)}$. En effet, l'organisation polycentrique du pays ne produit pas des disparités entre la capitale d'État et les régions, mais davantage entre l'Ouest et l'Est, entre anciens et nouveaux Länder. Les nouveaux Länder de l'Est jouissent donc de redistributions dites «horizontales » ${ }^{(74)}$ entre Länder et verticales via des subventions de l'État et la contribution des citoyens au travers du Solidaritätszuschlag (impôt de solidarité) mis en place en 1991. Le montant de ces redistributions fait l'objet d'évaluations divergentes, mais qui aboutissent toutes à des volumes colossaux ${ }^{(75)}$. La Loi fondamentale allemande de 1949,

72 Dufaux et al., « $\mathrm{n}^{\circ} 01$. Avis de naissance» (note 38).

73 Guy Baudelle, Klaus Kunzmann, «Regards croisés sur l’aménagement du territoire en France et en Allemagne», Espaces et sociétés, n 22 (2004), p. 69-77. 
par l'intermédiaire de son article $72^{(76)}$, appelle à une Einheitlichkeit der Lebensverhältnisse (unité des conditions de vie), l'État fédéral devant assurer les mêmes conditions de vie à tous. À partir de 1994, une modification de la loi ${ }^{(77)}$ prévoit que l'État pourvoit à des gleichwertige Lebensverhältnisse (conditions de vie équitables). Le changement terminologique qui intervient cette année-là avec le remplacement de Einheitlichkeit par gleichwertig ${ }^{(78)}$ peut être compris comme l'aveu d'une unité difficile à mettre en place, dans une Allemagne réunifiée depuis 4 ans au moment du changement de l'article 72 . Au tournant des années 2000 et jusqu'à la fin de cette décennie, des débats ${ }^{(79)}$ se déroulent au sujet de l'application de l'exigence d'équité des conditions de vie, dans un contexte de sous-équipement et de vieillissement des Kreise (arrondissements) ruraux des nouveaux Länder. L'État fédéral alimente lui-même ces débats grâce à la publication $^{(80)}$ en janvier 2015 du mensuel du ministère de l'Économie et des Énergies, consacré au bilan économique de la reconstruction des Länder de l'ancienne RDA. Le Ministère débute son rapport en affirmant que: "Hinsichtlich der Lebensqualität und Infrastruktur sind heute zwischen alten und neuen Ländern kaum mehr Unterschiede festzustellen ${ }^{(81)}$ (en ce qui concerne la qualité de vie et les infrastructures, les inégalités sont moindres entre les anciens et les nouveaux Länder), ce qui témoigne d'une progressive équité des conditions de vies entre anciens et nouveaux Länder.

Malgré les différences de configurations politiques et spatiales entre la France et l'Allemagne, on trouve quelques constantes dans la volonté du pouvoir central d'intégrer les territoires - et ainsi de corriger les disparités entre la capitale et la province en France, entre l'Ouest et l'Est en Allemagne. Dans les années 1960, la décision du

76 Article 72 (2) «Der Bund hat in diesem Bereich das Gesetzgebungsrecht, wenn und soweit ein Bedürfnis nach bundesgesetzlicher Regelung besteht, weil:

1. eine Angelegenheit durch die Gesetzgebung einzelner Länder nicht wirksam geregelt werden kann oder

2. die Regelung einer Angelegenheit durch ein Landesgesetz die Interessen anderer Länder oder die Gesamtheit beeinträchtigen könnte oder

3. die Herstellung der Rechts- oder Wirtschaftseinheit, insbesondere die Wahrung der Einheitlichkeit der Lebensverhältnisse im Bundesgebiet über das Gebiet eines Landes hinaus sie erfordert», Grundgesetz für die Bundesrepublik Deutschland, http://www.verfassungen.de/de/gg.htm, consulté le 19.12.2017.

77 Article 72 (2) «Auf der Gebieten des Artikels 74 Abs. 1 Nr. 4, 7, 11, 13, 15, 19a, 20, 22, 25 und 26 hat der Bund das Gesetzgebungsrecht, wenn und soweit die Herstellung gleichwertiger Lebensverhältnisse im Bundesgebiet oder die Wahrung der Rechts- oder Wirtschaftseinheit im gesamtstaatlichen Interesse eine bundesgesetzliche Regelung erforderlich macht», Grundgesetz für die Bundesrepublik Deutschland, https://www.gesetze-im-internet.de/gg/BJNR000010949.html, consulté le 19.12.2017.

78 Article 72 (note 77).

79 Eva Barlosius, "Gleichwertig ist nicht gleich», Politik und Zeitgeschichte (en ligne), $\mathrm{n}^{\circ} 37$ (2006), http://www.bpb.de/apuz/29548/gleichwertig-ist-nicht-gleich?p=all, consulté le 19.12.2017; Edmund BRAND, "Gleichwertige Lebensverhältnisse als Rechtsproblem », Berlin-Brandenburgische Akademie der Wissenschaften. Interdisziplinäre Arbeitsgruppe Zukunftsorientierte Nutzung ländlicher Räume, $\mathrm{n}^{\circ} 13$ (2006), $48 \mathrm{p}$.

80 Bundesministerium für Wirtschaft und Energie, «Herstellung gleichwertiger Lebensverhältnisse in ganz Deutschland - ein politischer Auftrag - Wirtschaftliche Entwicklung Ostdeutschlands 25 Jahre nach dem Mauerfall», Monatsbericht BMWI, n 1 (2015), 10 p.

81 Ibid., p. 1. 
ministre fédéral des Transports de RFA que «kein Deutscher soll mehr als 20 Kilometer von einer Autobahnauffahrt entfernt leben " ${ }^{(82)}$ (aucun citoyen allemand ne doit vivre à plus de $20 \mathrm{~km}$ d'une bretelle d'accès à l'autoroute) n'est pas sans rappeler le développement du réseau ferroviaire en France à la fin du XIX ${ }^{\mathrm{e}}$ siècle qui voulait optimiser les transports de la périphérie vers les bassins d'emploi. Ainsi, le Plan Freycinet de $1878^{(83)}$ prévoyait que toutes les sous-préfectures devaient être reliées au réseau de chemin de fer ainsi qu'un maximum de chefs-lieux de canton ${ }^{(84)}$. La justice spatiale prend ici la forme d'un accès égalitaire des citoyens aux moyens de transports et concourt à promouvoir un des leitmotivs de l'Union européenne: l'intégration territoriale.

Cette contextualisation en France et en Allemagne montre que les chronologies nationales et européennes ne coïncident pas: de part et d'autre du Rhin, la prise en compte du principe de justice précède la législation européenne des années 1990. La France et l'Allemagne, États fondateurs de l'Union européenne, déclinent à leur manière dans leurs pratiques d'aménagement un principe de cohésion devenu cardinal dans la politique communautaire. Ce principe apparaît dans le Traité sur l'Union européenne de 1992 qui affirme qu' "afin de promouvoir un développement harmonieux de l'ensemble de la Communauté, celle-ci développe et poursuit son action tendant au renforcement de sa cohésion économique et sociale» (article 1304 - Titre XIV) ${ }^{(85)}$. Il n'est alors question que de cohésion sociale et économique. La dimension territoriale est apparue plus tard dans les débats communautaires, sous influence française ${ }^{(86)}$. La cohésion territoriale est définie aujourd'hui par la Commission européenne à la fois comme un objectif et comme les moyens d'y parvenir. Développer la cohésion territoriale revient à valoriser la diversité des territoires et leurs atouts respectifs au service du développement de l'UE, limiter une excessive concentration urbaine, assurer une meilleure connexion des territoires entre eux de manière à ce que nul ne soit défavorisé dans l'accès aux services publics par son seul lieu de résidence, et enfin intensifier les coopérations inter-territoriales ${ }^{(87)}$. Depuis son apparition dans le vocabulaire de la politique régionale au début des années 1990, la cohésion a été institutionnalisée par son entrée dans le traité d'Amsterdam en 1997, confirmée dans le traité de Lisbonne (2007) et a pris un poids conséquent dans les équilibres budgétaires: la "cohésion» absorbe $34 \%$ des dépenses dans le budget communautaire entre 2014 et $2020^{(88)}$. Ainsi, bien que

82 Hahne/Stielike, "Gleichwertigkeit der Lebensverhältnisse» (note 29).

83 Loi du 17.07.1879.

84 Michel Aвнervé, «Le réseau de transport n'induit pas toujours du développement: illustration par le plan Freycinet pour les chemins de fer», Alternatives Économiques, blog, posté le 3.08.2014, http:// alternatives-economiques.fr/blogs/abherve/2014/08/03/le-reseau-de-transport-ninduit-pas-toujoursdu-developpement-illustration-par-le-plan-freycinet-pour-les-chemins-de-fer/, consulté le 19.12.2017. Commission européenne, Traité sur l'Union européenne, Maastricht, 1992.

86 Andréas FAludi, «La dimension territoriale de l'intégration européenne», L’information géographique, 71/4 (2010), p. 27-42; Nicolas GAUBERT, «La cohésion territoriale de l'Union européenne: un nouvel horizon de la justice spatiale? Retour sur le succès d'un terme», in: Dufaux/Philifert (dir.), Justice spatiale et politiques territoriales (note 24).

87 http://ec.europa.eu/regional_policy/fr/policy/what/territorial-cohesion/ consulté le 19.12.2017.

88 L’UE soutient par ailleurs la compétitivité et la croissance dans les territoires mieux dotés. En tout, la politique régionale absorbe donc $47 \%$ des dépenses communautaires entre 2014 et 2020, soit désormais davantage que l'agriculture. 
le projet européen ne se soit pas emparé frontalement de la notion de justice spatiale, il a produit des dispositifs et des concepts qui s'en approchent. Comment s'est réalisée cette évolution? En quoi peut-on relier la politique de cohésion territoriale et la justice spatiale?

Le préambule du traité de Rome (1957) promettait aux États membres de «renforcer l'unité de leurs économies et d'en assurer le développement harmonieux en réduisant l'écart entre les différentes régions et le retard des moins favorisées ». Mais l'aménagement du territoire est absent des compétences exclusives de l'Europe. Ce n'est d'ailleurs qu'en 1975 qu'a été créé le Fonds européen de développement régional (FEDER) dont les ressources, pendant plusieurs décennies, ont été distribuées par les États, et non selon une clé de répartition supranationale. L'Europe communautaire a donc pendant longtemps «réparé» les déséquilibres et désavantages territoriaux sans conceptualiser cette action, et en déléguant cette politique aux États. Ce n'est que dans les années 1990 qu'une réflexion théorique est venue étayer ces actions territoriales et qu'un cadrage conceptuel et opérationnel européen est apparu, au sein d'un groupe de réflexion prospective sur la relation entre la cohésion sociale et les territoires. Ces orientations théoriques ont été rassemblées dans un document de cadrage indicatif mais non contraignant envers les États, le Schéma de développement de l'espace communautaire (SDEC) ${ }^{(89)}$. Il est construit autour du «quasiconcept ${ }^{(90)}$ de cohésion, hybride car à la fois opérationnel et théorique, qui côtoie ceux de compétitivité et de développement durable ${ }^{(91)}$. La cohésion territoriale y est définie comme la «distribution géographiquement équilibrée de la croissance sur le territoire de l'UE» ${ }^{(92)}$. Les objectifs majeurs du SDEC sont un "développement spatial polycentrique», un accès «équitable» aux infrastructures et au savoir, et une gestion «prudente» du patrimoine naturel et culturel. En prévision des élargissements vers l'Est, les dépenses de cohésion ont ciblé prioritairement les régions dont le PIB par habitant est inférieur à $75 \%$ de la moyenne européenne, et l'éligibilité des régions aux dépenses de cohésion ou de compétitivité est élaborée au niveau européen, et non plus par les États.

La politique de cohésion, qui, depuis les années 2000, sous-tend les politiques régionales européennes, relève-t-elle d'une déclinaison de la justice spatiale? Les termes de justice ou d'équité sont absents du SDEC, qui leur préfère la notion d'équilibre. Mais la cohésion va au-delà de la politique régionale redistributive et réactive, qui fonctionnait depuis sa naissance avec le FEDER. En effet, elle se veut intégrée au cœur des décisions et des actions communautaires, y compris sectorielles, et prend acte du principe de différence cher à John Rawls ${ }^{(93)}$ : «L’un des traits décisifs de la politique de

89 Commission européenne, Schéma de développement de l'espace communautaire. Vers un développement spatial équilibré et durable du territoire de l'Union européenne, Luxembourg, Office des publications officielles des Communautés européennes, 1999, 89 p.

90 Paul BernARD, «La cohésion sociale: critique dialectique d'un quasi-concept», Lien social et Politiques, $\mathrm{n}^{\circ} 47$ (1999), p. 47-59.

91 N. GAubert, «La cohésion territoriale de l’Union européenne» (note 86).

92 Commission européenne (note 89).

93 Comme le rappelle la définition supra, au nom de la cohésion territoriale, la politique régionale communautaire promet de valoriser les singularités des territoires. 
cohésion est sa capacité à s'adapter aux besoins et aux caractéristiques spécifiques des territoires, en fonction des problèmes ou des opportunités résultant de leur situation géographique ${ }^{(94)}$; ce faisant, elle promeut un principe d'équité - et non d'égalité. Mais en tirant simultanément les fils de la cohésion, de la compétitivité et du développement durable, l'Europe elle aussi entretient la difficile tension entre performance et réparation, une tension explicitement exposée dans le SDEC ${ }^{(95)}$. Reste qu'empiriquement, $72 \%$ des dépenses régionales sont orientées vers les régions éligibles à l'objectif de cohésion, et $28 \%$ pour la compétitivité ${ }^{(96)}$ : on a bien privilégié la logique compensatrice.

\section{Conclusion}

La notion de justice spatiale, au caractère volatile, permet un éclairage ou une relecture des différences, des disparités, des discontinuités socio-spatiales. Elle échappe à une lecture univoque de part et d'autre du Rhin mais également au sein d'un même champ disciplinaire, de même que son ancrage conceptuel est largement hérité des travaux anglo-saxons du côté allemand alors que des géographes français la théorisent dès les années 1970. Comme le montre l'analyse chronologique des acceptions de la notion de justice spatiale, ainsi que ses déclinaisons empiriques, son actualité scientifique dans la géographie française et allemande a permis de renouveler essentiellement la géographie urbaine, rurale, politique, environnementale, sans toutefois aboutir à une construction théorique commune. L'application différenciée de la notion de justice spatiale à une lecture des inégalités s'avère également orientée par les contextes historiques et géographiques nationaux, ce dont témoigne particulièrement le cas allemand.

Par ailleurs, la justice spatiale est principalement étudiée sous l'angle des injustices et/ou des inégalités, que la géographie et l'aménagement tentent de comprendre à plusieurs échelles, parfois dans le but de les corriger. La notion de justice spatiale et ses multiples acceptions servent une recherche-action structurée par l'analyse et la dénonciation d'inégalités et d'injustices, et expriment également une volonté de replacer le chercheur dans une posture non surplombante, plus engagée. Du côté de l'aménagement, les termes de la justice sont supposés inspirer des politiques volontaristes d'action: il s'agit de compenser des déséquilibres, de développer des territoires délaissés ou marginalisés. C'est ainsi que l’Union européenne envisage de contribuer à une meilleure justice spatiale sans pourtant utiliser l'expression. Toutefois, l'abondante littérature critique sur la "cohésion» européenne s'avère finalement assez peu sécante avec celle qui est consacrée à la justice spatiale, laquelle a davantage investi des terrains extra-européens et des échelles micro.

94 Commission des communautés européennes, Une politique de cohésion pour soutenir la croissance et l'emploi. Orientations stratégiques communautaires 2007-2013, Bruxelles, Communication de la Commission, 2005, 43 p., ici p. 33.

95 «À cet effet, il est important de faire concorder les objectifs du développement, de l'équilibre et de la conservation. Une politique exclusivement orientée vers l'équilibre entraînerait l'affaiblissement des régions fortes sur le plan économique, et renforcerait parallèlement la dépendance des régions moins favorisées. Le développement seul favoriserait l'accroissement des disparités régionales» (SDEC, 1999, p. 11). 


\title{
Résumé
}

Entendue comme un outil de lecture des inégalités socio-spatiales, la notion de justice spatiale pose la question de la justice en géographie et en aménagement. Si la géographie marxiste l'interroge depuis les années 1970, des travaux scientifiques récents renouvellent les approches, notamment en France et en Allemagne. Ce terme recouvre une multitude d'objets de recherche et de grilles de lecture des espaces et des territoires. Comment s'est structuré son champ? Cet article propose une comparaison des acceptions, des usages ainsi que des évolutions de la notion de justice spatiale entre France et Allemagne pour en analyser l'application dans le domaine des disparités territoriales et régionales en France, en Allemagne et dans l'Union européenne. L'article revient sur les évolutions de la problématique de la justice spatiale dans les écrits scientifiques au regard des contextes politiques des deux pays (1); il passe ensuite en revue différentes acceptions et usages de la notion dans la littérature géographique (2), avant d'examiner comment le principe de justice spatiale a inspiré les politiques françaises, allemandes et européennes d'aménagement du territoire (3).

\section{Zusammenfassung}

Die "räumliche Gerechtigkeit" betrifft die Frage der Gerechtigkeit in der Geographie und Raumplanung und kann als Hilfe in der Interpretation der räumlichen und sozialen Ungleichheiten angesehen werden. Während die marxistische Geographie diese Vorstellung seit den Siebzigerjahren hinterfragt, erneuern neue wissenschaftliche Arbeiten diese Ansätze, besonders in Frankreich und Deutschland. Die „räumliche Gerechtigkeit“ umfasst eine Vielfalt an Forschungsobjekten und Bewertungsmatrix von Räumen und Territorien. Wie war sein Feld strukturiert? Der hier präsentierte Artikel schlägt einen Blick über die Bedeutung seiner räumlichen Übersetzung in der französischen und deutschen Geographie vor. Wir stellen hier einen diachronischen Ansatz der räumlichen Gerechtigkeit unter ihrem begrifflichen und methodologischen Rahmen vor (1), mit Parallelen ihrer empirischen Deklination in dem geographischen Bereich (2). Schließlich werden wir über die Operationalisierung des Begriffs auf den französischen, deutschen und europäischen Ebenen der Raumplanung diskutieren (3).

\begin{abstract}
Understood as a key of reading socio-spatial inequalities, Spatial Justice raises the issue of Justice in the fields of Geography and Land Planning. Although the Marxist Geography has been questioning it since the 1970s, recent scientific studies illustrate new approaches especially in France and Germany. In fact, Spatial Justice covers several research topics and reading grids of spaces and territories. How was this structured field of research? This paper provides a comparative view on its meanings and spatial translations in French and German geography and also an analysis of its application in the field of territorial disparities in France, Germany and in the European Union. A diachronic approach of Spatial Justice (1) is presented in light of its theoretical and methodological frameworks just like its empirical variations (2). Finally this article proposes a discussion about its operational effectiveness at the French, German and European level of Land Planning (3).
\end{abstract}

